\title{
Computer-aided protocol analysis of writing processes
}

\author{
C. MICHAEL LEVY \\ University of Florida, Gainesville, Florida \\ and \\ SARAH RANSDELL \\ Florida Atlantic University, Davie, Florida
}

\begin{abstract}
With increased interest in process analysis of writing has come an expanded role for computers. Records of written and verbal protocols are made by capturing keystrokes and speech, playing them back on videotape, and then coding, second by second, the duration, frequency, and effort of various writing subprocesses as they unfold. In this paper we introduce a new approach to analyzing think-aloud and resource allocation protocols in writing research and describe how analyses of them can add to current models of writing.
\end{abstract}

For more than a decade, the think-aloud protocol analysis devised by Hayes and Flower (1980) has been the dominant tool for obtaining algorithmic-level data about the processes involved in written composition. The procedure involves asking subjects to "think aloud" as they conduct some primary task (Ericsson \& Simon, 1984, 1993). An alternative technique has measured writing processes by calling upon the subject to do directed retrospection-that is, to stop writing and identify the process in which they were just engaged (Kellogg, 1987, 1988).

In this paper we describe a new approach to analyzing the processes involved in writing. We believe that a process orientation will facilitate the development of explanatory models of writing and complement the think-aloud and directed retrospective techniques already in use. In the following sections we describe several traditional frameworks for studying writing processes. We then describe our approach and how we believe it can enhance del development in writing research. Finally, we offer some conclusions about the potential benefits to theory development in writing research that stem from our process-oriented analysis.

\section{Model Development in Writing Research}

The most widely cited model of writing processes is Hayes and Flower's (1980) description, which is based on analysis of the contents of think-aloud protocols. The main components of Hayes and Flower's model focus on three basic writing processes: planning, generating text, and revising. Bereiter and Scardamalia (1987) have proposed a more detailed pair of models of writing (knowledge-

Correspondence may be sent to either author: C. M. Levy, Department of Psychology, University of Florida, Gainesville, FL 32611 (e-mail: levy@psych.ufl.edu), or S. Ransdell, 2912 College Ave. Florida Atlantic University, Davie, FL 33314 (e-mail: ransdell@acc.fau.edu). telling and knowledge-transforming), but their source of data has also been mainly limited to the contents of thinkaloud protocols. The development of models of writing has been relatively slow due to the lack of fundamental information about how these processes interact through time.

On-line time and effort information can support theory development by validating the categories of data created from think-aloud protocol analysis. In our research, the categories generated by observing sample data add empirical validation, because Hayes and Flower's (1980) three writing subprocesses characterize nearly all the activities in which the writer engages during a writing task. Now that basic subprocesses have been identified, we need to specify the relative distribution of time and effort that is allocated to them within a writing session and across many writing sessions. Computer-supported records of on-line writing processes facilitate this task.

Kellogg $(1987,1988)$ began the task of categorizing the time and effort of writing processes through the method of directed retrospection, in which writers are interrupted periodically and are asked to identify which of the three tasks they are employing. Directed retrospection provides detailed time course and effort information about writing processes, but it is potentially more intrusive than recording behaviors directly in real time. Other differences between our approach and directed retrospection are more substantive. For example, our categorization is empirically rather than theoretically derived. Kellogg distinguishes between planning and reviewing processes (and asks his writers to choose between the two), but our process analyses suggest that the two cannot be easily distinguished on the basis of think-aloud protocols or writing behavior itself. For example, revision is reliably indicated by making adjustments to text that has already been generated. Reviewing is not, since it could be categorized as planning future content or reviewing past writing. To 
delineate each process more clearly, we coordinate the information available from verbal protocols with writing behavior.

\section{Product-Oriented Protocol Analysis}

Protocol analysis is widely used to generate hypotheses about mental processes during writing (see Swarts, Flower, \& Hayes, 1984). This method involves transcribing speech and organizing it into segments, which are then coded for various content categories. The method has only recently been used for hypothesis-testing purposes, however, in part because of the difficulty in managing the large amounts of data available (but see Crutcher, Ericsson, \& Wichura, 1994, and Sanderson, McNeese, \& Zaff, 1994, in this issue). Furthermore, these large amounts of data about the contents of protocols need to be supplemented by equally detailed process information.

Anderson (1987) has argued that a low ratio of data to theoretical detail will hamper scientific induction about mental processing. Protocols offer a way to improve this ratio; they provide a state-by-state description of what a subject pays attention to and is able to report from working memory. Protocols are not complete traces of mental processing, but they do provide a subset of the thoughts heeded while completing a task. Arguments concerning the reactivity and validity of protocols have largely been resolved (see Ericsson \& Simon, 1993, for their latest coverage of these issues). More recently, the problem of using protocols in theory development is one of managing this large source of data and providing converging evidence from other behavioral data sources.

Computer-aided analysis of the contents of protocols has proven to be a useful and efficient system for dealing with the large amounts of data obtained (for a review, see James \& Sanderson, 1991). These product-oriented programs filter and organize the contents of protocols to aid heuristic analyses. They also support the statistical analysis of content and patterns (sequential data analysis) in protocol data. The present approach, highlighted in the following section, supplements product analysis.

\section{Process-Oriented Protocol Analysis}

Process-oriented protocol analysis focuses on identifying the processes that occur during writing as these processes unfold in real time. In the present approach, in which writers are sometimes asked to perform a secondary task such as thinking aloud, and a tertiary task such as responding quickly to a tone, we took inexpensive, offthe-shelf materials and wrote a special-purpose code (Levy, Fryman, \& Ransdell, 1994) to develop an integrated system. Our writers compose on IBM-compatible 486 computers in a Windows environment, which permits several programs to run concurrently. As Figure 1 shows, the typical screen layout provides an area occupying about three fourths of the screen for the writer to use in composing a document. More complex word processors can be successfully used in this panel, but for our purposes we wanted the subjects to use word-processing

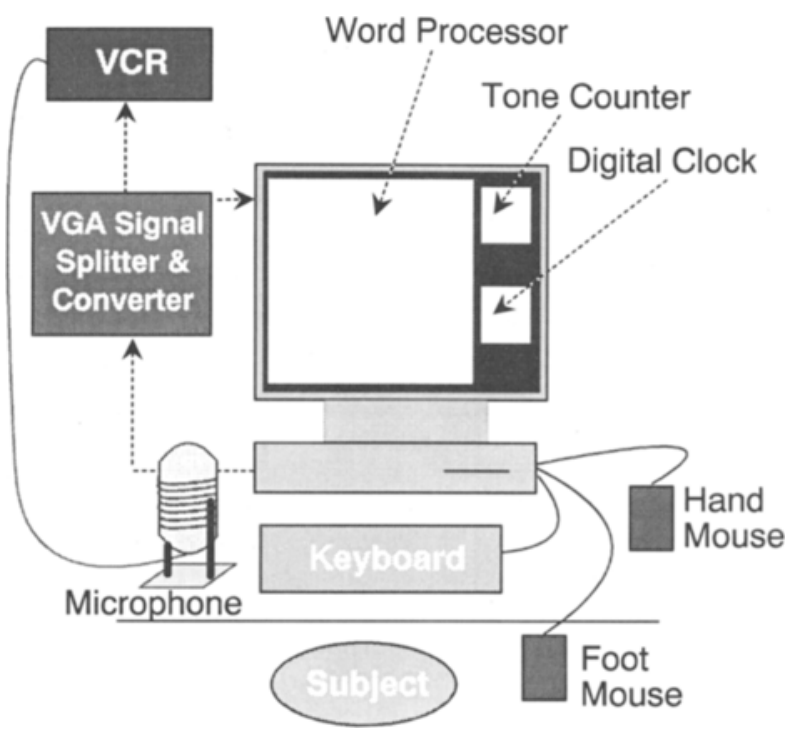

Figure 1. Visual schematic of equipment configuration.

software that included only the most basic features. Thus, our writers used Notepad, a program bundled with Windows software.

Ransdell and Levy (in press) describe writing protocols collected with a technique dependent on a special-purpose terminate-and-stay resident (TSR) program that records on disk the time when each key is depressed, and which permits replaying of this record later in real time. This TSR program works effectively with character-based (but not graphics-based) word-processing software. Because it is not feasible to perform the equivalent of fast forward, reverse, or still-frame operations common on videotape recorders using this software, it is extremely cumbersome to use.

Our first enhancement to the TSR methodology was to abandon the TSR program altogether, replacing it with a videotape recording of each writing session. This was made possible by an off-the-shelf interface that converts the VGA signal provided by the computer for the monitor into an NTSC (or PAL) broadcast-quality signal that is fed directly into a video recorder (VCR). For our work, we chose the Simulscan because it exhibited no discernible degradation of the signal on the monitor and no slowing of the image due to scan rate. It was also inexpensive (about $\$ 500$ ), partly because it supported only the $640 \times 480$ VGA mode used by current Windows applications. ${ }^{1}$ Thinkaloud protocols are easily recorded simultaneously via a microphone connected to the VCR.

When it is important to include an additional task, such as to respond quickly to a tone presented while the subject is both writing and thinking aloud, we use a program called BEEPER, which has been developed expressly for this purpose (Levy et al., 1994). BEEPER runs in the Windows environment as a background task while a writer is using a word processor (or any other Windows application). It sounds a tone aperiodically (in our case, a 30-sec variable interval) and examines a COMM port that sig- 
nals when the writer has depressed a button on a secondary mouse. Since the writer's hands are usually engaged with the keyboard keys or primary mouse, this secondary mouse is placed on the floor to enable its use as a foot switch.

Once the data are collected, both the recorded visual and auditory signals are played back in real time to enable raters to code the duration and frequency of various writing subprocesses (planning and reviewing, generating text, and revising). Videotapes of word-processed text, as well as simultaneous audio records of think-aloud protocols, can be abstracted without the need for manual verbatim transcription. We use EventLog (Henderson, 1989), a computer emulation of an $\mathrm{n}$-channel event recorder, to code behaviors that relate to the writing subprocesses. Raters watch a replay of a writing session on a TV monitor and simply match the behavior they see unfold with operationally defined keypresses. EventLog provides frequency and duration distributions of selected channel behaviors over a specified time frame and temporal resolution. We then process the ASCII file that EventLog produces through a locally developed program that categorizes the writer's behavior on a second-by-second basis.

Interrater agreement is critical to valid judgments of time on task. Raters can be trained within a few hours to high levels of reliability for such categories as typing or text generation, pausing at various boundaries, changing the text, and commenting on the topic or task itself. Table 1 shows the behaviors subsumed into the coding of each writing process for the written and verbal protocol categories. A clear benefit of collecting both keystrokes in the written protocol and speech in the verbal protocol is that the two records can be used to discriminate difficult categories. For example, while pausing in generating text, a writer may say that he/she is planning what to write next. In this case, the verbal protocol can

Table 1

Coding Categories Corresponding to Three Main Writing Processes

Coding Categories for Written Protocols

Typing (GT)

Deleting (R)

Superficial errors $(R)$

Meaningful changes (R)

Pausing within a word $(\mathrm{P} / \mathrm{R})$

Pausing within a clause or sentence $(P / R)$

Pausing within a paragraph $(\mathrm{P} / \mathrm{R})$

General pausing or between paragraphs $(\mathbf{P} / \mathbf{R})$

Any movement in text $(\mathrm{P} / \mathrm{R})$

New paragraph (P/R)

Coding Categories for Verbal Protocols

Pausing in speech (P/R)

Writing content (GT)

Speaking and writing the same content (GT)

Planning future topic content $(\mathbf{P} / \mathbf{R})$

Rereading text written $(\mathbf{P} / \mathbf{R})$

Note-Generating text (GT), revising $(\mathrm{R})$, and planning/reviewing $(\mathrm{P} / \mathrm{R})$ indicate how each EventLog category corresponds to Hayes and Flower's (1980) three main writing processes. be reliably coded as planning, even in the absence of any observable writing behavior.

The data from a recent study (Ransdell \& Levy, 1993) illustrate high levels of reliability between coders. For example, the categories of planning, generating text, and revising yielded interrater reliabilities of $.97, .99$, and .94 , respectively. These interrater reliabilities were calculated across the total times for 3 raters, who scored each of three writing subprocesses for 10 accomplished writers, who composed for 10 experimental sessions. Interrater reliabilities calculated in this manner, however, can overestimate the accord between raters. Consider, for example, a hypothetical 40-min writing record. Rater A maintains that planning occurred during the first $20 \mathrm{~min}$ and that generating text and revising occupied the balance of the session. In Rater B's coding, all planning occurred during the last $20 \mathrm{~min}$. Since both raters determined that $20 \mathrm{~min}$ were devoted to planning, the researcher should be pleased with their consistency. If they were similarly in accord as they examined other records, the interrater reliabilities would approach unity. As this example should make clear, however, the raters really never agreed at all on what behavior or process was occurring at any particular moment. An index that can determine the extent to which raters agree in their codings over an extended period for a specific response category is needed. We developed a special-purpose program, AGREE, to determine this index.

AGREE does a fine-grained comparison of the coding protocols of a pair of raters. In our use, it examines each second of their EventLog tracing. If the raters agree at time $T$ that the writer exhibited behavior $X$, that fact is noted. In contrast, if Rater A scored the behavior as $X$ and Rater B scored it as $Y$, the program randomly determines that one of the raters made the correct judgment and assigns his/her rating to the protocol for that moment in the record. It also notes the discord, incrementing two counters-one that notes that Rater A's coding behavior $X$ is disputed, and a second that similarly increments the discord over Rater B's coding of behavior $Y$. For each writing process, the program calculates an agreement score by determining the ratio of the total number of seconds that both raters agreed about a process to the total number of seconds that either rater indicated that the process occurred. The ratio is then expressed as a percentage agreement. ${ }^{2}$ Values for agreement between raters on planning $(92.2 \%)$, generating text $(69.9 \%)$, and revising (30.7\%) are a more conservative estimate than interrater reliability.

Figure 2 illustrates the granularity of the data that can be studied after this type of processing. This level of analysis can reveal behaviors not noticeable in analyses that provide less temporal density, or focus exclusively on content. To obtain these data, a writer closed her eyes during the initial 2-min baseline and depressed the secondary mouse with her foot whenever she heard the tone. She was then given a topic about which she was to write dur- 


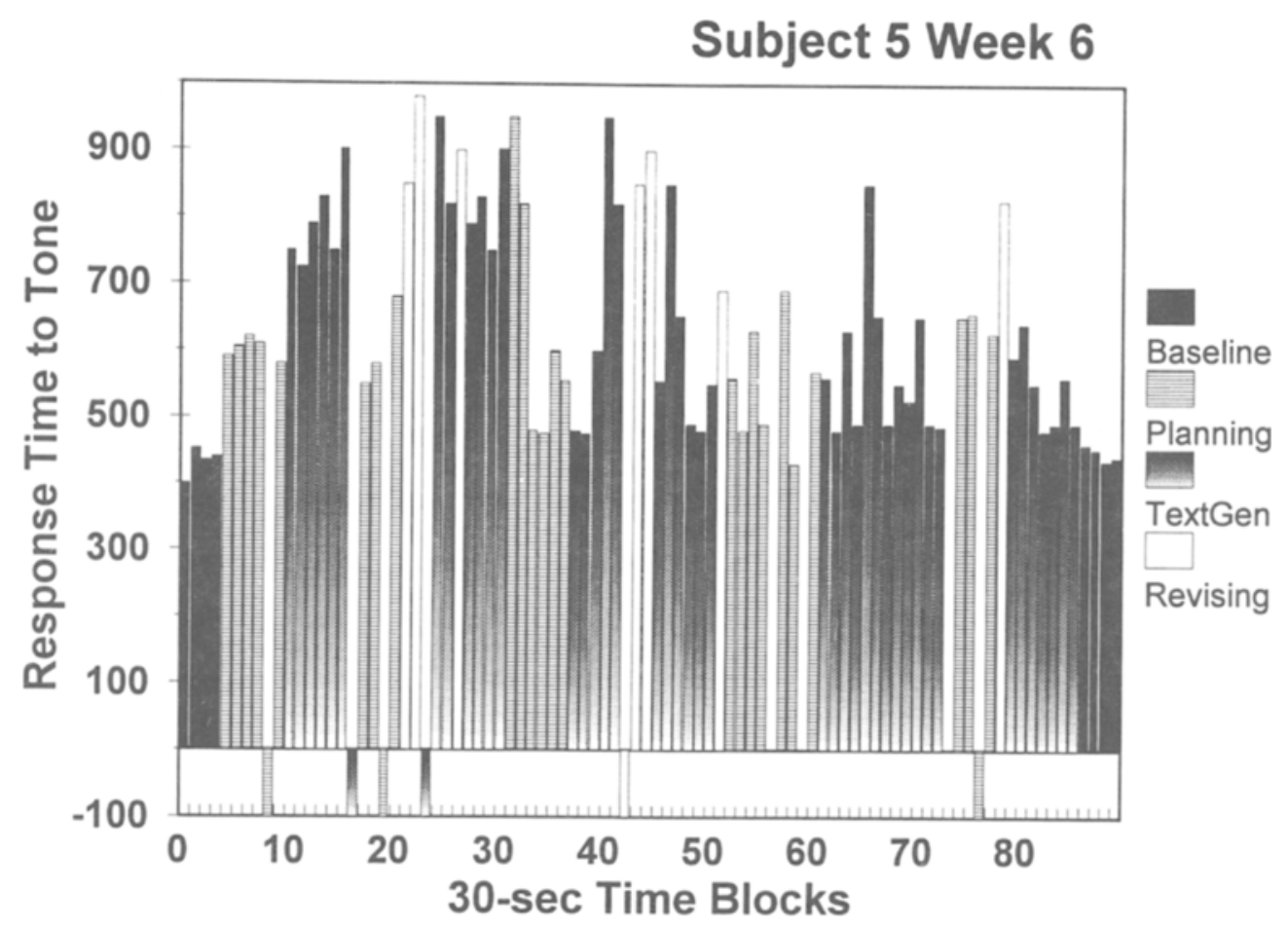

Figure 2. Sample data showing reaction time to tone as a function of baseline, writing subprocesses, and $30-$ sec blocks within a writing session.

ing the next $40 \mathrm{~min}$. She had been instructed to think aloud while writing and also to continue responding to the tone whenever she heard it. During the final $2 \mathrm{~min}$ of the session, she again closed her eyes and responded only to the tone.

Once the videotape of her session was scored by raters using EventLog, their ratings were processed by AGREE, which defined the writing process that occurred during each second of the protocol. These definitions were examined in relation to the BEEPER record, which stored the onset and response times for each tone. In Figure 2, response times exceeding $3 \mathrm{sec}$ were defined as misses; these are shown in the graph as lines extending below the baseline. With such a fine-grained portrayal of the data, it is possible to almost continuously track the subject's allocation of resources to various writing subprocesses within a session. For example, notice in Figure 2 that within the first $20 \mathrm{sec}$ of writing the writer shifted between planning and text generation twice, and that in this period response time nearly doubles for generating text, as opposed to planning.

\section{Conclusions}

Our methods are complementary to the type of content analyses that have been used previously. They do, however, provide unique information about the relative frequency and duration of various writing subprocesses. Theory development in writing research needs a greater number of reliable methods for uncovering cognitive pro- cesses if this field is to progress as others have done. For example, research on the processes involved in reading has greatly benefited from the converging evidence provided by on-line records of protocol data used in conjunction with behavioral data and predictions from formal theory (Magliano \& Graesser, 1991; Olson, Duffy, \& Mack, 1984; Suh \& Trabasso, 1993). This so-called "threepronged" approach can be successfully implemented in the area of writing research. Because word-processed writing is already a part of a computer record, the data can be gathered with minimal intrusion to the natural task. Computer-based recordings of the type we describe, such as second-by-second tracking of resource allocation across time and writing subprocess, provide information that has previously not been available.

Such data may change how we study writing; such data may also alter our views of how writers compose. Without this detailed process information, models of writing will be slow to proceed from their current descriptive emphasis. Current models of writing were predominately developed by analyzing the products or contents of thinkaloud protocols. This paper has highlighted a way to use these protocols to better understand the time course and effort of writing processes as well. Temporally dense process information is sorely needed in written composition research, and there has been only limited progress (see Matsuhashi, 1982). Microcomputers greatly facilitate obtaining minimally intrusive process data from protocols, and such information is critical to support model development in writing research. 


\section{REFERENCES}

Anderson, J. R. (1987). Methodologies for studying human knowledge. Behavioral \& Brain Sciences, 10, 467-505.

BereIter, C., \& SCARDAmalia, M. (1987). The psychology of written composition. Hillsdale, NJ: Erlbaum.

Crutcher, R. J., Ericsson, K. A., \& Wichura, C. A. (1994). Improving the encoding of verbal reports using MPAS: A computeraided encoding system. Behavior Research Methods, Instruments, \& Computers, 26, 167-171.

Ericsson, K. A., \& Simon, H. A. (1984). Protocol analysis: Verbal reports as data. Cambridge, MA: MIT Press.

Ericsson, K. A., \& Simon, H. A. (1993). Protocol analysis: Verbal reports as data (rev. ed.). Cambridge, MA: MIT Press.

HAYES, J. R., \& FLOWER, L. (1980). Identifying the organization of writing processes. In L. W. Gregg \& E. R. Steinberg (Eds.), Cognitive processes in writing (pp. 31-50). Hillsdale, NJ: Erlbaum.

Henderson, R. (1989). EventLog. Iowa City: Conduit.

James, J. M., \& SANDERson, P. M. (1991). Heuristic and statistical support for protocol analysis with SHAPA Version 2.01. Behavior Research Methods, Instruments, \& Computers, 23, 449-460.

KELLOGG, R. T. (1987). Effects of topic knowledge on the allocation of processing time and cognitive effort to writing processes. Memory \& Cognition, 15, 256-266.

KELLOGG, R. T. (1988). Attentional overload and writing performance: Effects of rough draft and outline strategies. Journal of Experimental Psychology: Learning, Memory, \& Cognition, 14, 355-365.

Levy, C. M., Fryman, J. B., \& Ransdell, S. E. (1994). Measuring response latency while running Windows applications programs. Manuscript in preparation.

Magliano, J. P., \& Graesser, A. C. (1991). A three-pronged method for studying inference generation in literary text. Poetics, 20, 193-232.

MatsuHASHI, A. (1982). Explorations in the real-time production of written discourse. In M. Nystrand (Ed.), What writers know: The language, process, and structure of written discourse (pp. 269-290). New York: Academic Press.

Olson, G. M., Duffy, S. A., \& MaCk, R. L. (1984). Thinking-outloud as a method for studying real-time comprehension processes. In D. Kieras \& M. Just (Eds.), New methods in the study of immediate processes in comprehension (pp. 165-187). Hillsdale, NJ: Erlbaum.
RansDell, S. E., \& Levy, C. M. (1993, November). A longitudinal process approach to understanding writing. Paper presented at the meeting of the Psychonomic Society, Washington, DC.

RANSDElL, S. E., \& LeVY, C. M. (in press). Writing as process and product: The impact of tool, genre, audience knowledge and writer expertise. Computers in Human Behavior.

Sanderson, P. M., McNeese, M. D., \& ZafF, B. S. (1994). Handling complex real-world data with two cognitive engineering tools: COGENT and MacSHAPA. Behavior Research Methods, Instruments, \& Computers, 26, 117-124.

SUH, S., \& Trabasso, T. (1993). Inferences during reading: Converg. ing evidence from discourse analysis, talk-aloud protocols, and recognition priming. Journal of Memory \& Language, 32, 279-300.

Swarts, H., Flower, L., \& HaYes, J. R. (1984). Designing protocol studies of the writing process: An introduction. In R. Beach \& L. Bridwell (Eds.), New directions in composition research (pp. 53-71). New York: Guilford.

\section{NOTES}

1. This particular device may be discontinued by the time this article is published, but there are many comparable devices.

2. Actually, AGREE does considerably more work than this in order to calculate its index. When it examines the EventLog record of each rater, it refers to the time that the rater began scoring and to the time after that, when the rater scored the first response from the VCR. The time between these two events depends on uncontrolled factors, such as when the rater started on the VCR. Briefly, the program aligns in the following way. First, it determines which rater first started coding a given writing session and uses this datum as a fixed referent. Next, it provisionally realigns the records by linking the start times of the raters and calculates an agreement index as described above. Subsequently, it adjusts the second rater's time of origin back $1 \mathrm{sec}$, then recalculates the agreement index. It repeats this process until it has reduced the second rater's origin time by 2 min. Finally, it determines which of the 120 calculated offsets resulted in the maximum overall agreement between raters and it uses that value to render the final scoring decisions. 